\title{
Public awareness and willingness to use automated external defibrillators in a metropolitan city
}

\author{
Jae Hyuk Lee', Dong Eun Lee ${ }^{2}$, Hyun Wook Ryoo', Sungbae Moon', \\ Jae Wan Cho', Yun Jeong Kim², Jong Kun Kim², Jung Ho Kim³, \\ Kyung Woo Lee ${ }^{4}$, Sang-chan Jin ${ }^{5}$, Jun Seok Seo ${ }^{6}$
}

\begin{abstract}
Department of Emergency Medicine, ${ }^{1}$ Kyungpook National University Hospital, Kyungpook National University School of Medicine, Daegu; ${ }^{2}$ Kyungpook National University Chilgok Hospital, Kyungpook National University School of Medicine, Daegu; ${ }^{3}$ Yeungnam University College of Medicine, Daegu; ${ }^{4}$ Catholic University of Daegu School of Medicine, Daegu; ${ }^{5}$ Keimyung University Dongsan Medical Center, Keimyung University School of Medicine, Daegu; ${ }^{6}$ Dongguk University Ilsan Hospital, Dongguk University College of Medicine, Seoul, Korea
\end{abstract}

Objective Early defibrillation is crucial for the survival of patients with out-of-hospital cardiac arrest. This study aimed to examine the trends and associated factors regarding public awareness and willingness to use automated external defibrillators (AEDs) through citywide surveys.

Methods Three-round surveys were conducted in February $2012(n=1,000)$, December 2016 $(n=1,141)$, and December $2018(n=1,001)$ among citizens in Daegu, South Korea, who were aged $\geq 19$ years. The subjects were selected through a three-stage quota sampling. Awareness and willingness to use an AED were assessed in the three groups. The primary outcome was willingness to use AEDs.

Results Of 3,142 respondents, 3,069 were eligible for analysis. The proportion of respondents who knew how to use AEDs increased from 4.7\% in 2012 to 20.8\% in 2018. Of the respondents in 2012, 2016, and 2018, 39.7\%, 50.0\%, and 43.2\%, respectively, were willing to use an AED. Factors associated with willingness to use AEDs were male sex (adjusted odds ratio [AOR], 1.39; 95\% confidence interval $[\mathrm{Cl}], 1.10-1.75)$, cardiopulmonary resuscitation training experience in the previous 2 years (AOR, 1.80;95\% Cl, 1.43-2.28), recognition of the Good Samaritan law $\left(A O R, 1.45 ; 95 \% \mathrm{Cl}_{1} 1.13-1.86\right)$, and awareness of how to use an AED (AOR, 4.40; 95\% Cl, 3.265.93).

Conclusion To increase willingness to use AEDs, education in AED use and the Good Samaritan law, along with re-education to maintain knowledge of AED use, should be considered.

Keywords Defibrillators; Cardiopulmonary resuscitation; Surveys and questionnaires
elSSN: 2383-4625

Received: 19 May 2020

Revised: 28 June 2020

Accepted: 29 June 2020

Correspondence to: Dong Eun Lee Department of Emergency Medicine, Kyungpook National University School of Medicine, 680 Gukchaebosang-ro, Jung-gu, Daegu 41944, Korea

E-mail: delee@knu.ac.kr ORCID

https://orcid.org/0000-0002-2057-5261

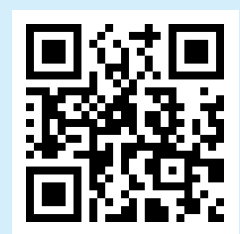

How to cite this article:

Lee JH, Lee DE, Ryoo HW, Moon S, Cho JW, Kim YJ, Kim JK, Kim JH, Lee KW, Jin SC, Seo JS. Public awareness and willingness to use automated external defibrillators in a metropolitan city. Clin Exp Emerg Med 2021;8(1):1-8. https://doi.org/10.15441/ ceem. 20.053

This is an Open Access article distributed under the terms of the Creative Commons Attribution Non-Commercial License (https:// creativecommons.org/licenses/by-nc/4.0/). 


Wapsule $\begin{aligned} & \text { What is already known } \\ & \text { Known factors associated with willingness to use automated external defibrillators (AEDs) were male sex, witnessing a } \\ & \text { cardiac arrest, prior cardiopulmonary resuscitation training experience, prior AED training, and actual experience in us- } \\ & \text { ing AEDs. } \\ & \text { What is new in the current study } \\ & \text { Recognition of the Good Samaritan law and awareness of how to use an AED are associated with willingness to use } \\ & \text { AEDs. To increase willingness to use AEDs, education in AED use and the Good Samaritan law, along with re-education } \\ & \text { to maintain knowledge of AED use, should be considered. }\end{aligned}$

\section{INTRODUCTION}

Out-of-hospital cardiac arrests (OHCAs) are a major burden on public health. According to previous studies, the incidence of OHCA of cardiac origin in adults is 54.6 per 100,000 person-years worldwide. $^{1,2}$ In patients with $\mathrm{OHCA}$, early defibrillation is crucial for survival. ${ }^{3-5}$ Kitamura et al. reported that nationwide installation of public access automated external defibrillators (AEDs) in Japan increased the rate of defibrillation performed by laypersons and thereby improved the survival outcomes among patients after OHCA. ${ }^{6}$ Furthermore, a link between emergency dispatch centers and first responders and the implementation of public access defibrillation (PAD) programs were also associated with improvements in survival by contributing to prompt defibrillation. ${ }^{7-9}$

Since 2008, legislation involving both school law for mandatory cardiopulmonary resuscitation (CPR) training and PAD law in a public facility has been passed in South Korea. Lee et al. reported that changes in nationwide CPR policies may have affected the citizens' awareness and willingness to perform bystander CPR. ${ }^{10}$ According to a report by the Korea Centers for Disease Control and Prevention, the proportion of bystander performed CPRs actually increased from $1.9 \%$ in 2008 to $21.0 \%$ in $2017 .^{11}$ However, the proportion of defibrillations using public AEDs remains approximately $0.7 \%$, which is significantly lower than those in other countries such as the United States, Japan, and European countries, where the proportions range between $2.5 \%$ and 16\%., $82-15$

Therefore, it is necessary to evaluate the barriers to the use of AEDs, considering that the actual use of AEDs is low even though approximately 45,000 AEDs are currently installed in South Korea. ${ }^{16}$ Since 2012, Daegu Metropolitan City, South Korea, and the Daegu Emergency Medical Cooperation Committee have been conducting three-round surveys on citizens' awareness of CPR and AEDs. Through the three-round surveys, we examined how citizens' perceptions and willingness to use AEDs changed over time. We aimed to examine the trend of awareness and willingness to use AEDs through citywide surveys. We also investigated the factors associated with the willingness to use AEDs.

\section{METHODS}

\section{Study setting, design, and subjects}

Daegu Metropolitan City has a population of approximately 2.48 million and an area of $883.54 \mathrm{~km}^{2}$. The incidence of OHCAs (those presumed to have medical causes) per 100,000 people was 35.7 in 2012, which increased to 41.7 in 2017. ${ }^{11}$ The Emergency Medical Services Act of 2008 required the installation of AEDs in apartments with $\geq 500$ households and in public facilities such as airports, railway stations, trains, ships, and public offices. Since then, the distribution of AEDs has constantly been increasing in South Korea. The overall number of public AEDs in Daegu, excluding those deployed in medical facilities and by emergency medical services, was 203 in 2014 and increased to 979 in $2018 .^{16}$

In this cross-sectional study, we analyzed data from threeround surveys in Daegu. The surveys were conducted in February $2012(n=1,000)$, December $2016(n=1,141)$, and December 2018 $(n=1,001)$ among Daegu citizens aged $\geq 19$ years. The subjects were selected through a three-stage quota sampling based on age, sex, and district population distribution, according to population census data. Quota sampling is a method of non-probability sampling when the samples are selected based on the probability proportionate to the distribution of a variable in the population. ${ }^{17}$ This sampling method is used to ensure that the proportion of samples for each category (such as sex or age group) will have the same proportion assumed to exist in the population. The selected target population in each survey was different from, and had no relation to, the previous survey population. In each survey, before conducting each survey, we informed all participants about the purpose and objective of the surveys and obtained their 
written consent. The interviewers visited each respondent at his or her home to conduct face-to-face interviews using structured questionnaires. ${ }^{18,19}$ Before the surveys were conducted, meetings were held to standardize interview methods and explain the questionnaires and detailed guidelines, as described in previous studies. $^{18,19}$

\section{Questionnaires and variables}

Emergency physicians drafted and revised the questionnaires for each survey. The questionnaire administered in 2018 is shown in Supplementary Material 1. The following information was obtained from the questionnaires: (1) demographic data (i.e., sex, age, educational status, household income); (2) attitude toward and willingness to perform CPR; (3) awareness of the Good Samaritan law; (4) prior CPR training experience (i.e., training type, last training time, and number of training sessions attended); and (5) awareness and willingness to use AEDs. Some respondents did not answer questions about CPR training experience, willingness to perform $C P R$, confidence in the ability to perform CPR, and awareness and willingness to use AEDs, and so those variables were excluded from the analysis (Fig. 1). The primary outcome was willingness to use AEDs.

\section{Statistical analysis}

Respondents were divided into three groups according to the date of the survey. The distribution of demographic data and of variables regarding confidence and willingness to perform CPR, prior CPR training, and awareness and willingness to use AEDs was analyzed according to the study group. Pearson's chi-square test and the extended Mantel-Haenszel chi-square test for linear trends were carried out to assess the significance of differences among the three groups. Logistic regression analysis was performed to investigate the association between the study group and the outcome. We included potential confounding variables such as age, sex, educational status, and household income. To evaluate factors associated with willingness to use AEDs, we also performed a multiple logistic regression analysis. Factors selected from the univariate analysis $(P<0.05)$ were included as independent variables in a logistic regression model using a stepwise method (Supplementary Material 2). To determine whether the logistic model fit the data, we calculated the Hosmer-Lemeshow goodness of fit. The results were calculated as odds ratios (ORs) and 95\% confidence intervals (Cls). SAS ver. 9.4 (SAS Institute Inc., Cary, NC, USA) and MedCalc ver. 19.2.1 (MedCalc Software, Ostend, Belgium) were used to perform the statistical tests. Pvalues of less than 0.05 were considered statistically significant.

\section{Ethics statement}

This study was approved by the institutional review board of Kyungpook National University Chilgok Hospital (2020-03-038). The requirement for informed consent was waived owing to the retrospective nature of this study.

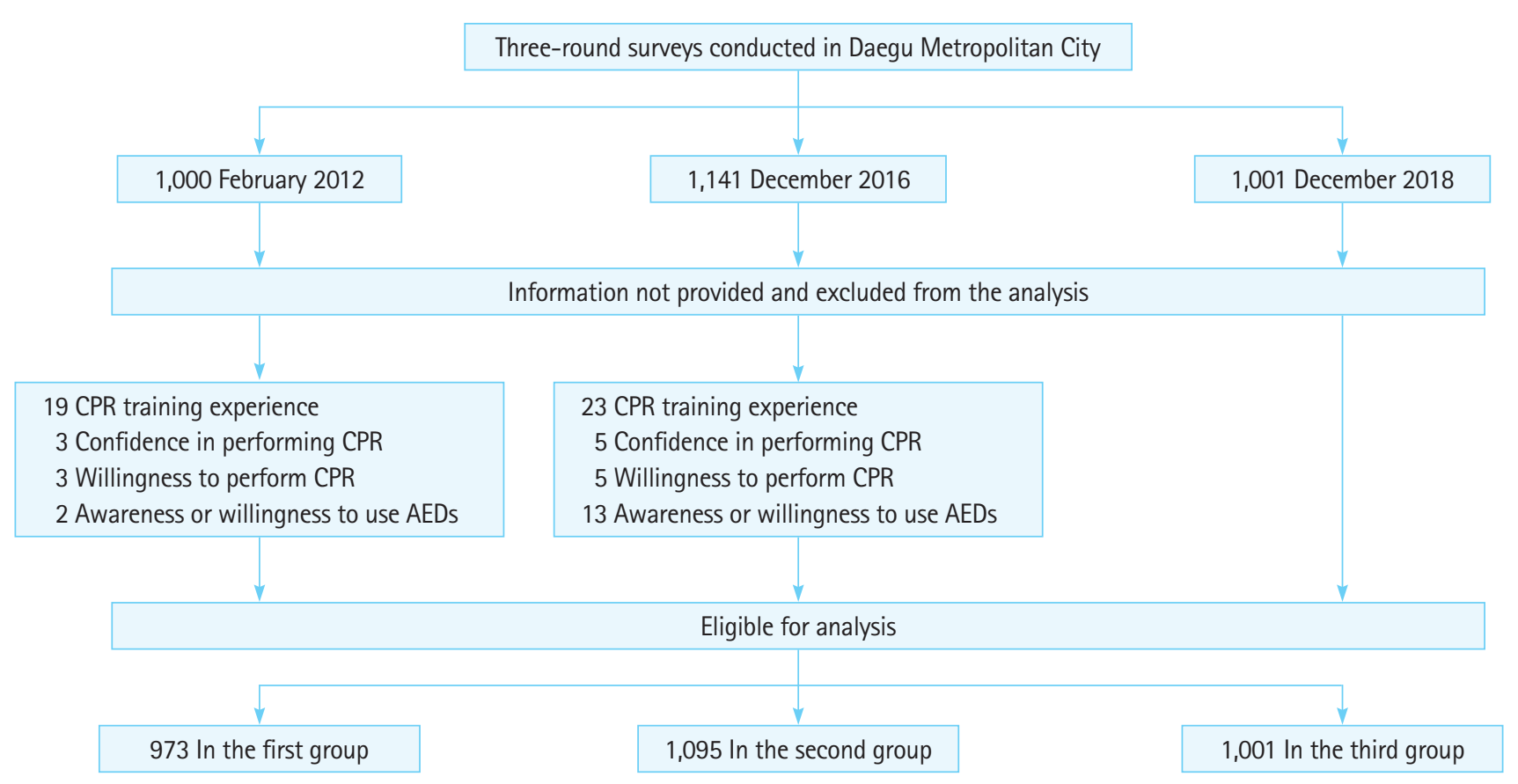

Fig. 1. Study flow diagram. AED, automated external defibrillator; CPR, cardiopulmonary resuscitation. 


\section{RESULTS}

\section{Characteristics of respondents}

A total of 3,142 people responded to the surveys conducted in 2012, 2016, and 2018. Among them, 73 respondents were excluded due to incomplete data. Therefore, a total of 3,069 respondents were included in the analysis: 973 in the 2012 survey (first group), 1,095 in the 2016 survey (second group), and 1,001 in the 2018 survey (third group) (Fig. 1). No difference in sex distributions was observed. The proportion of respondents aged $\geq 60$ years increased over time, as follows: $19.8 \%$ in the first group, $22.7 \%$ in the second group, and $24.1 \%$ in the third group (Table 1). The proportion of respondents who recognized the Good Samaritan law increased from $10.2 \%$ in the first group to $33.9 \%$ in the third group ( $P$ for trend $<0.001$ ).

\section{Prior CPR training experience}

The percentage of respondents with prior CPR training experience was $36.1 \%$ in the first group, whereas that in the second and third groups was 55.8\% and 54.7\%, respectively. The proportion of respondents who had received prior CPR training within the previous 2 years was 17.4\%, 31\%, and 29.8\% in the first, second, and third groups, respectively. Meanwhile, the percentage of respondents who had undergone AED training during the CPR training course was 5.1\%, 26.6\%, and $25.9 \%$ in the first, second, and third groups, respectively (Table 2 ).

\section{Awareness and willingness to use AEDs}

The percentage of respondents who were aware of what an AED is increased from $37.1 \%$ in the first group to $82.7 \%$ in the third group ( $\mathrm{P}$ for trend $<0.001$ ). The proportion of respondents who were willing to use AEDs was 39.7\%, 50.0\%, and 43.2\% in the first, second, and third groups, respectively ( $P$ for trend $=0.129$ ) (Fig. 2). After adjustment for confounding variables, the adjusted ORs (AORs) for willingness to use an AED were $1.54\left(95 \% \mathrm{Cl}_{\text {, }}\right.$ 1.28-1.85) for the second group and $1.19(95 \% \mathrm{Cl}, 0.98-1.44)$ for the third group (Table 3 ).

\section{Factors associated with willingness to use AEDs}

The factors associated with willingness to use AEDs were male sex (AOR, 1.39; 95\% Cl, 1.10-1.75), CPR training experience in the previous 2 years $(A O R, 1.80 ; 95 \% \mathrm{Cl}, 1.43-2.28)$, recognition

Table 1. General characteristics of respondents

\begin{tabular}{|c|c|c|c|c|c|}
\hline Variable & Total & First group & Second group & Third group & P-value \\
\hline Sex & & & & & 0.797 \\
\hline Male & 1,452 & $463(47.6)$ & $524(47.9)$ & $465(46.5)$ & \\
\hline Female & 1,617 & $510(52.4)$ & $571(52.1)$ & $536(53.5)$ & \\
\hline Age (yr) & & & & & 0.061 \\
\hline$<60$ & 2,388 & $781(80.3)$ & $847(77.4)$ & 760 (75.9) & \\
\hline$\geq 60$ & 681 & $192(19.7)$ & $248(22.6)$ & $241(24.1)$ & \\
\hline Education & & & & & $<0.001$ \\
\hline No response & 26 & $10(1.0)$ & $0(0.0)$ & $16(1.60)$ & \\
\hline High school graduate or under & 1,125 & $414(42.5)$ & $379(34.6)$ & $332(33.2)$ & \\
\hline Undergraduate or higher & 1,918 & $549(56.4)$ & $716(65.4)$ & $653(65.2)$ & \\
\hline Household income (KRW) & & & & & $<0.001$ \\
\hline No response & 170 & $39(4.0)$ & $55(5)$ & $76(7.60)$ & \\
\hline$<2 \mathrm{M}$ & 876 & $346(35.6)$ & $316(28.9)$ & $214(21.4)$ & \\
\hline $2-4 \mathrm{M}$ & 1,253 & $439(45.1)$ & $461(42.1)$ & $353(35.3)$ & \\
\hline$>4 \mathrm{M}$ & 770 & $149(15.3)$ & $263(24)$ & $358(35.8)$ & \\
\hline \multicolumn{6}{|l|}{ Family history of heart disease } \\
\hline Yes & 365 & $69(7.10)$ & $150(13.7)$ & $146(14.6)$ & $<0.001$ \\
\hline \multicolumn{6}{|c|}{ Awareness of the Good Samaritan law } \\
\hline Yes & 785 & $99(10.2)$ & $347(31.7)$ & $339(33.9)$ & $<0.001$ \\
\hline \multicolumn{6}{|l|}{ Confidence in ability to perform CPR } \\
\hline Yes & 1,257 & $328(33.7)$ & $497(45.4)$ & $432(43.2)$ & $<0.001$ \\
\hline \multicolumn{6}{|l|}{ Willingness to perform CPR } \\
\hline Yes & 1,256 & $529(54.4)$ & $386(35.3)$ & $341(34.1)$ & $<0.001$ \\
\hline \multicolumn{6}{|l|}{ AED awareness } \\
\hline I have seen public AEDs & 1,540 & $162(16.6)$ & $711(64.9)$ & $667(66.6)$ & $<0.001$ \\
\hline
\end{tabular}

KRW, Korean won; M, million; CPR, cardiopulmonary resuscitation; AED, automated external defibrillator. 
Jae Hyuk Lee, et al.

Table 2. Comparison of CPR training experience among respondents

\begin{tabular}{|c|c|c|c|c|c|}
\hline Variable & Total & First group & Second group & Third group & P-value \\
\hline \multicolumn{6}{|l|}{ CPR training } \\
\hline Yes & 1,510 & $351(36.1)$ & $611(55.8)$ & $548(54.7)$ & $<0.001$ \\
\hline No. of CPR training sessions & & & & & 0.992 \\
\hline 1 & 518 & $118(12.1)$ & $209(19.1)$ & $191(19.1)$ & \\
\hline 2 & 512 & $118(12.1)$ & $209(19.1)$ & $185(18.5)$ & \\
\hline$\geq 3$ & 480 & $115(11.8)$ & $193(17.6)$ & $172(17.2)$ & \\
\hline Time since the most recent CPR training & & & & & 0.076 \\
\hline$<2 \mathrm{yr}$ & 806 & $169(17.4)$ & $339(31.0)$ & $298(29.8)$ & \\
\hline$\geq 2 \mathrm{yr}$ & 704 & $182(18.7)$ & $272(24.8)$ & $250(25.0)$ & \\
\hline \multicolumn{6}{|l|}{ AED training included } \\
\hline Yes & 600 & $50(5.1)$ & $291(26.6)$ & $259(25.9)$ & $<0.001$ \\
\hline
\end{tabular}

Values are presented as number or number (\%).

CPR, cardiopulmonary resuscitation; AED, automated external defibrillator.

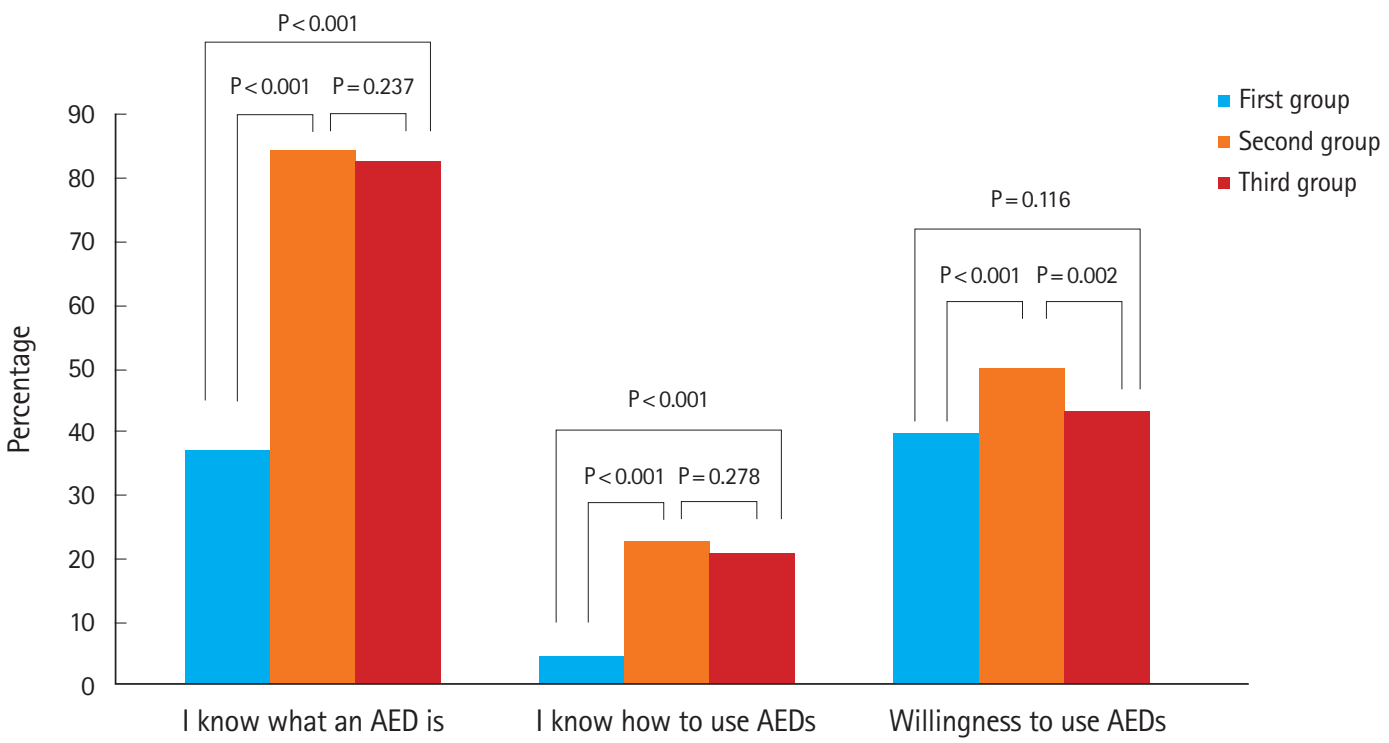

Fig. 2. Comparison of awareness and willingness to use automated external defibrillators (AEDs) according to study group.

Table 3. Multiple logistic regression analysis of the study outcome (willingness to use AED) according to respondent group

\begin{tabular}{lcccc}
\hline Group & No. & $\%$ & AOR & $95 \% \mathrm{Cl}$ \\
\hline First group & 386 & 39.5 & 1.00 & - \\
Second group & 548 & 50.0 & 1.54 & $1.28-1.85$ \\
Third group & 432 & 43.2 & 1.19 & $0.98-1.44$ \\
\hline
\end{tabular}

The first group comprised respondents to the 2012 survey, the second group to the 2016 survey, and the third group to the 2018 survey. Results were adjusted for sex, age, family history of heart disease, degree of education, and income. $A E D$, automated external defibrillator; $A O R$, adjusted odds ratio; $\mathrm{Cl}$, confidence interval.

of the Good Samaritan law (AOR, 1.45; 95\% Cl, 1.13-1.86), and awareness of how to use an AED (AOR, 4.40; 95\% Cl, 3.26-5.93) in the final logistic regression stepwise model, as shown in Fig. 3.

\section{DISCUSSION}

In this study, the proportion of respondents who were aware of what an AED is, who knew how to use it, and who were willing to use it was larger in 2016 than in 2012. However, we observed no difference between the proportions of respondents in 2016 and 2018 who were aware of what an AED is and how to use it. Moreover, the willingness to use AEDs decreased in 2018 compared to 2016. To our knowledge, this is the first study to evaluate regional trends in the awareness and willingness to use AEDs among citizens in South Korea.

In South Korea, the Emergency Medical Services Act has been amended to reflect mandatory CPR training and deployment of AEDs. As a result, the number of public AEDs in Daegu Metropoli$\tan$ City increased to 39.8 per 100,000 people in $2018,{ }^{20}$ and the 


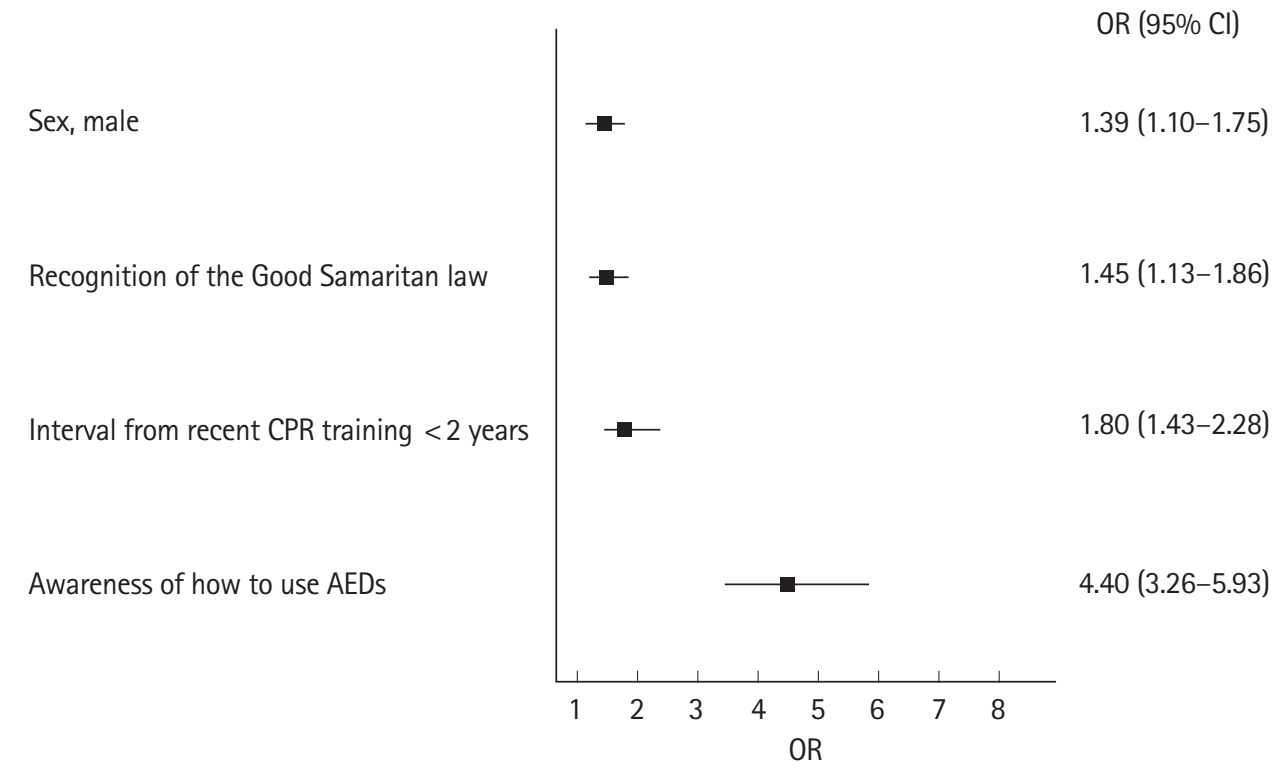

Fig. 3. Factors associated with willingness to use automated external defibrillators (AEDs). Forest plot shows the odds ratio (OR) and 95\% confidence interval (CI) for each factor, calculated in the final stepwise logistic regression models. Sex, age, educational status, monthly income, confidence in ability to perform cardiopulmonary resuscitation (CPR), AED awareness, prior CPR training experience, and recognition of the Good Samaritan law were included as independent variables.

proportion of respondents who reported seeing public AEDs also increased in this study. Furthermore, in this survey, we observed that the proportion of respondents who had prior CPR training experience and AED training as part of CPR education was higher in 2016 and 2018 than in 2012. Thus, there is a possibility that the increase in prior CPR training experience and number of public AED accounted for the increase in respondents indicating "I know what an AED is" and "I know how to use an AED." On the other hand, the proportion of respondents who were willing to use AEDs increased from 39.7\% in 2012 to 50.5\% in 2016, but then decreased to $43.2 \%$ in 2018. The increase in the percentage of respondents who had previous CPR experience within the past 2 years and AED training or who were aware of the Good Samaritan law might have contributed to the increase in the willingness to use AEDs in 2016. However, in 2018, a decreasing pattern in the willingness to use AEDs was observed, accompanied by a plateau in the rate of previous CPR training experience among respondents. CPR education in the regional community and dissemination of public information regarding AEDs might have been insufficient to increase the willingness of citizens to use AEDs between 2016 and 2018.

In a survey of 2,084 citizens in the United Kingdom, the factors associated with willingness to use AEDs were male sex, witnessing a cardiac arrest, prior CPR training experience, prior AED training, and actual experience in using AEDs. ${ }^{21}$ These findings are similar to our findings that male sex, prior CPR training experience within the previous 2 years, and awareness of how to use AEDs were associated with willingness to use AEDs. For the responders to this survey, not knowing how to use AEDs was the most common barrier to the use of AEDs, followed by concerns about harming the patient and concerns about legal responsibility. In addition, the proportion of people who do not know how to use AEDs has decreased simultaneously with the increasing proportion of people concerned about legal responsibility regarding their use (Supplementary Material 3). Although the proportion of respondents who indicated that they did not know how to use AEDs decreased, not knowing how to use AEDs remained as the most common barrier to the use of AEDs. Thus, it is important that every CPR education course should include AED practical training to increase AED use. Moreover, the Korean Good Samaritan law is a provision for reducing criminal liability in the event of a patient's death. ${ }^{22}$ The Emergency Medical Services Act, therefore, needs to be revised in the direction of exempting liability to reduce concerns about legal liability among those who are willing to help.

Son et al. ${ }^{19}$ reported that re-education within a certain timeframe following CPR education appears to be necessary because the willingness of bystanders to perform CPR tends to decrease over time. Prior CPR training experience within the last 2 years is also associated with willingness to use AEDs. Therefore, a decrease in the knowledge of AED use may reduce the bystander's willingness to use AEDs. These findings emphasize the importance of re-educating people on AED use to maintain their knowledge 
on the subject. The recognition of the Good Samaritan law is also associated with the willingness to use AEDs. In particular, Moon et al. ${ }^{18}$ found an association between the recognition of the Good Samaritan law and the willingness to perform CPR on a stranger. However, in our study, only $33.9 \%$ of respondents were aware of the Good Samaritan law in 2018. Meanwhile, Schober et al. ${ }^{22}$ reported that large-scale public information campaigns to improve awareness of AEDs are important after AEDs are deployed. Therefore, these findings suggest that CPR education, public information campaigns on AEDs, and education about the Good Samaritan law should be provided to improve the use of public AEDs.

This study has several limitations. First, the survey itself could have been influenced by selection bias. To minimize selection bias, we used a quota sampling method. Second, among respondents who were willing to use an AED, their responses to the survey may differ from actual performance and willingness to use an AED in a real OHCA situation. In a study conducted in the Netherlands, only $6 \%$ of respondents mentioned AEDs when asked about what they would do if they witnessed a suspected sudden cardiac arrest. ${ }^{23}$ The respondents' confidence in their ability, or their willingness, to perform CPR or use AEDs could have been overestimated in comparison with their actual capacity to do so; this is a disadvantage of surveys. Although the willingness to use AEDs increased to 50\% in 2016, the actual use of public AEDs remained minimal. It is helpful to prepare a community intervention program to link increased willingness to actual use. Lastly, this survey relied on the respondents' memories; therefore, there could have been differences between the time they answered and the actual time and number of their prior CPR and AED training experience, making their responses subject to recall bias.

In summary, this study revealed that the willingness to use AEDs among citizens in Daegu increased from 2012 to 2016 but decreased from 2016 to 2018. We also found that the factors associated with the willingness to use AEDs included male sex, prior CPR training, recognition of the Good Samaritan law, and awareness of how to use an AED. Thus, to increase willingness to use AEDs, it would be essential to train more people on AED use and educate them about the Good Samaritan law. Re-education should also be considered for designated first responders to maintain their knowledge and willingness to use AEDs.

\section{CONFLICT OF INTEREST}

No potential conflict of interest relevant to this article was reported.

\section{SUPPLEMENTARY MATERIAL}

Supplementary Materials are available from: https://doi.org/10.15441/ ceem.20.053.

Supplementary Material 1. Questionnaire for the survey conducted in 2018

Supplementary Material 2. Univariate analysis of willingness to use automated external defibrillators

Supplementary Material 3. Barriers to the use of AEDs

\section{REFERENCES}

1. Berdowski J, Berg RA, Tijssen JG, Koster RW. Global incidences of out-of-hospital cardiac arrest and survival rates: systematic review of 67 prospective studies. Resuscitation 2010;81:147987.

2. Myat A, Song KJ, Rea T. Out-of-hospital cardiac arrest: current concepts. Lancet 2018;391:970-9.

3. Hazinski MF, Idris AH, Kerber RE, et al. Lay rescuer automated external defibrillator ("public access defibrillation") programs: lessons learned from an international multicenter trial: advisory statement from the American Heart Association Emergency Cardiovascular Committee; the Council on Cardiopulmonary, Perioperative, and Critical Care; and the Council on Clinical Cardiology. Circulation 2005;111:3336-40.

4. Hallstrom AP, Ornato JP, Weisfeldt M, et al. Public-access defibrillation and survival after out-of-hospital cardiac arrest. N Engl J Med 2004;351:637-46.

5. Song KJ, Kim JB, Kim J, et al. Part 2. Adult basic life support: 2015 Korean Guidelines for Cardiopulmonary Resuscitation. Clin Exp Emerg Med 2016;3(Suppl):S10-6.

6. Kitamura T, Iwami T, Kawamura T, et al. Nationwide public-access defibrillation in Japan. N Engl J Med 2010;362:994-1004.

7. Ringh $M$, Rosenqvist $M$, Hollenberg J, et al. Mobile-phone dispatch of laypersons for CPR in out-of-hospital cardiac arrest. N Engl J Med 2015;372:2316-25.

8. Ringh $M$, Jonsson $M$, Nordberg $P$, et al. Survival after public access defibrillation in Stockholm, Sweden: a striking success. Resuscitation 2015;91:1-7.

9. Pijls RW, Nelemans PJ, Rahel BM, Gorgels AP. A text message alert system for trained volunteers improves out-of-hospital cardiac arrest survival. Resuscitation 2016;105:182-7.

10. Lee MJ, Hwang SO, Cha KC, Cho GC, Yang HJ, Rho TH. Influence of nationwide policy on citizens' awareness and willingness to perform bystander cardiopulmonary resuscitation. Resuscitation 2013;84:889-94.

11. Korea Centers for Disease Control and Prevention. 2006-2017 
Sudden cardiac arrest statistics report [Internet]. Cheongju: Korea Centers for Disease Control and Prevention; 2018 [cited 2020 Mar 28]. Available from: http://www.cdc.go.kr/board.es? $\mathrm{mid}=$ a20503050000\&bid $=0021$ \&act $=$ view\&list_no $=142010$.

12. Ro YS, Song KJ, Shin SD, et al. Association between countylevel cardiopulmonary resuscitation training and changes in survival outcomes after out-of-hospital cardiac arrest over 5years: a multilevel analysis. Resuscitation 2019;139:291-8.

13. Chan PS, McNally B, Tang F, Kellermann A; CARES Surveillance Group. Recent trends in survival from out-of-hospital cardiac arrest in the United States. Circulation 2014;130:1876-82.

14. Kitamura T, Kiyohara K, Sakai T, et al. Public-access defibrillation and out-of-hospital cardiac arrest in Japan. N Engl J Med 2016;375:1649-59.

15. Agerskov M, Nielsen AM, Hansen $C M$, et al. Public access defibrillation: great benefit and potential but infrequently used. Resuscitation 2015;96:53-8.

16. National Emergency Medical Center. Emergency medical statistics yearbook [Internet]. Seoul: National Emergency Medical Center; 2019 [cited 2020 Mar 28]. Available from: https:// www.e-gen.or.kr/nemc/statistics_annual_report.do.

17. Rukmana D. Quota sampling. In: Michalos AC, editor. Encyclopedia of quality of life and well-being research. Dordrecht:
Springer Netherlands; 2014. p. 5382-4.

18. Moon S, Ryoo HW, Ahn JY, et al. A 5-year change of knowledge and willingness by sampled respondents to perform bystander cardiopulmonary resuscitation in a metropolitan city. PLoS One 2019;14:e0211804.

19. Son JW, Ryoo HW, Moon S, et al. Association between public cardiopulmonary resuscitation education and the willingness to perform bystander cardiopulmonary resuscitation: a metropolitan citywide survey. Clin Exp Emerg Med 2017;4:80-7.

20. National Emergency Medical Center. Emergency medical s tistics yearbook [Internet]. Seoul: National Emergency Medical Center; 2018 [cited 2020 Mar 28]. Available from: https:// www.e-gen.or.kr/nemc/statistics_annual_report.do.

21. Hawkes CA, Brown TP, Booth $S$, et al. Attitudes to cardiopulmonary resuscitation and defibrillator use: a survey of UK adults in 2017. J Am Heart Assoc 2019;8:e008267.

22. Korean Legislation Research Institute. Article 5-2 of the Emergency Medical Service Act amended on August 4, 2011. Available from: https://elaw.klri.re.kr/eng_service/lawView.do?hseq $=50784$ ctlang $=$ ENG.

23. Schober P, van Dehn FB, Bierens JJ, Loer SA, Schwarte LA. Public access defibrillation: time to access the public. Ann Emerg Med 2011;58:240-7. 